\title{
A Characterization of Saturated Designs for Factorial Experiments
}

\author{
Roberto Fontana \\ Department of Mathematical Sciences, Politecnico di Torino \\ Fabio Rapallo \\ Department DISIT, Università del Piemonte Orientale \\ Maria Piera Rogantin \\ Department of Mathematics, Università di Genova
}

\begin{abstract}
In this paper we study saturated fractions of factorial designs under the perspective of Algebraic Statistics. We define a criterion to check whether a fraction is saturated or not with respect to a given model. The proposed criterion is based purely on combinatorial objects. Our technique is particularly useful when several fractions are needed. We also show how to generate random saturated fractions with given projections, by applying the theory of Markov bases for contingency tables.

Keywords: Estimability; Linear model; Circuits; Graver basis; Universal Markov basis.
\end{abstract}

\section{Introduction}

The search for minimal designs to estimate linear models is an active research area in the design of experiments. Given a model, saturated fractions have a minimum number of points to estimate all the parameters of a given model. As a consequence, all information is used to estimate the parameters and there is no degree of freedom to estimate the error term. Nevertheless, saturated fractions are of common use in sciences and engineering, and they become particularly useful for highly expensive experiments, or when time limitations impose the choice of the minimum possible number of design points. For general reference in the design of experiments, the reader can refer to [19] and [3], where the issue of saturated fractions is discussed. 
In this paper we characterize saturated fractions of a factorial design in terms of the circuits of their design matrices and we define a criterion to check whether a given fraction is saturated or not. This avoids computating the determinant of the corresponding design matrix.

Our work falls within the framework of Algebraic statistics. The application of polynomial algebra to the design of experiments was originally presented in [17, but with a different point of view. The techniques used here are mainly based on the combinatorial and algebraic objects associated to the design matrix of the model, such as the circuits, the Graver basis and the Universal Gröbner basis. These algebraic objects are different bases of the toric ideal of the design matrix, which are a special set of polynomials originally used in Statistics for the analysis contingency tables. All these bases are used to solve enumeration problems, to make non-asymptotic inference, and to describe the geometric structure of the statistical models for discrete data. A recent account of this theory can be found in [10].

In this paper, we benefit from the interplay between algebraic techniques for the analysis of contingency tables and some topics of the design of experiments. We identify a factorial design with a binary contingency table whose entries are the indicator function of the fraction, i.e., they are equal to 1 for the fraction points and 0 for the other points. This implies that a fraction can also be considered as a subset of cells of the table. Some recent results in this direction can be found in [1]. The connections between experimental designs and contingency tables have also been explored in [11, but were limited to the investigation of enumerative problems in the special cases of contingency tables arising from the Sudoku problems.

The basic idea underlying our theory is as follows. A fraction is saturated if a null linear combination of its points with non-zero coefficients exists. This vector of coefficients belongs to the kernel of the transpose of the design matrix. IN algebraic language this means generating bases of the toric ideal associated to the design matrix. Thus, each null linear combination with integer coefficients translates into a binomial of the toric ideal of the design matrix. In this paper we prove that if we consider a special basis of the toric ideal, namely the circuit basis, this is also a sufficient condition. Our approach based on circuits avoids the computation of the determinant of the design matrix, and therefore it avoids possible numerical problems. It is particularly useful when we need to find several saturated fractions for the same model as in the case of algorithms for optimal design generation. Furthermore, the circuits can be computed once and for all from the design matrix of the full factorial model and do not depend on the fraction.

The paper is organized along these lines. In Section 2 we set some 
notations and we state the problem. In Section 3 we provide the basic algebraic framework to be used in the paper. In Section 4 we prove the main result, showing that the absence of circuits is a necessary and sufficient condition for obtaining a saturated fraction. In Section 5] for unimodular design matrices, we report results which highlight the relationship between the different bases of the relevant toric ideal, and we show that for several models the design matrix is unimodular. In Section 6 we provide some examples to demonstrate the practical applicability of our theory. In Section [7 we show how to generate a sample of saturated fractions, by applying the theory of Markov bases when we add constraints to the projections. Finally, in Section 8 we suggest some future research directions stimulated by the theory presented here.

\section{Saturated designs}

Let $\mathcal{D}$ be a full factorial design with $d$ factors, $A_{1}, \ldots, A_{d}$ with $s_{1}, \ldots, s_{d}$ levels respectively, $\mathcal{D}=\left\{0, \ldots, s_{1}-1\right\} \times \cdots \times\left\{0, \ldots, s_{d}-1\right\}$. We consider a linear model on $\mathcal{D}$ :

$$
Y=X \beta+\varepsilon,
$$

where $Y$ is the response variable, $X$ is the design matrix, $\beta$ is the vector of model parameters, and $\varepsilon$ is a vector of random variables that represent the error terms. We denote by $p$ the number of estimable parameters.

For instance, in a two-factor design with the simple effect model, we have $p=s_{1}+s_{2}-1$ and a possible design matrix is:

$$
X=\left(m_{0}\left|a_{0}\right| \ldots\left|a_{s_{1}-2}\right| b_{0}|\ldots| b_{s_{2}-2}\right),
$$

where $m_{0}$ is a column vector of 1 's, $a_{0}, \ldots, a_{s_{1}-2}$ are the indicator vectors of the first $\left(s_{1}-1\right)$ levels of the factor $A_{1}$, and $b_{0}, \ldots, b_{s_{2}-2}$ are the indicator vectors of the first $\left(s_{2}-1\right)$ levels of the factor $A_{2}$.

A subset $\mathcal{F}$, or fraction, of a full design $\mathcal{D}$, with minimal cardinality $\# \mathcal{F}=p$, that allows us to estimate the model parameters, is a saturated fraction or saturated design. By definition, the design matrix $X_{\mathcal{F}}$ of a saturated design is a non-singular matrix with dimensions $p \times p$.

Example 2.1. Let us consider the $2^{4}$ design and the model with simple effects and 2-way interactions. This example is at the same time not trivial and easy to handle, so that we will use it as the running example in this paper. The design matrix $X$ of the full design has rank equal to 11 and is reported in Figure 1. As the matrix $X$ has rank 11, we search for fractions 


$\left.\begin{array}{cccccccccccc} & 1 & a_{0} & b_{0} & c_{0} & d_{0} & a_{0} b_{0} & a_{0} c_{0} & a_{0} d_{0} & b_{0} c_{0} & b_{0} d_{0} & c_{0} d_{0} \\ (0,0,0,0) & 1 & 1 & 1 & 1 & 1 & 1 & 1 & 1 & 1 & 1 & 1 \\ (0,0,0,1) & 1 & 1 & 1 & 1 & 0 & 1 & 1 & 0 & 1 & 0 & 0 \\ (0,0,1,0) & 1 & 1 & 1 & 0 & 1 & 1 & 0 & 1 & 0 & 1 & 0 \\ (0,0,1,1) & 1 & 1 & 1 & 0 & 0 & 1 & 0 & 0 & 0 & 0 & 0 \\ (0,1,0,0) & 1 & 1 & 0 & 1 & 1 & 0 & 1 & 1 & 0 & 0 & 1 \\ (0,1,0,1) & 1 & 1 & 0 & 1 & 0 & 0 & 1 & 0 & 0 & 0 & 0 \\ (0,1,1,0) & 1 & 1 & 0 & 0 & 1 & 0 & 0 & 1 & 0 & 0 & 0 \\ (0,1,1,1) & 1 & 1 & 0 & 0 & 0 & 0 & 0 & 0 & 0 & 0 & 0 \\ (1,0,0,0) & 1 & 0 & 1 & 1 & 1 & 0 & 0 & 0 & 1 & 1 & 1 \\ (1,0,0,1) & 1 & 0 & 1 & 1 & 0 & 0 & 0 & 0 & 1 & 0 & 0 \\ (1,0,1,0) & 1 & 0 & 1 & 0 & 1 & 0 & 0 & 0 & 0 & 1 & 0 \\ (1,0,1,1) & 1 & 0 & 1 & 0 & 0 & 0 & 0 & 0 & 0 & 0 & 0 \\ (1,1,0,0) & 1 & 0 & 0 & 1 & 1 & 0 & 0 & 0 & 0 & 0 & 1 \\ (1,1,0,1) & 1 & 0 & 0 & 1 & 0 & 0 & 0 & 0 & 0 & 0 & 0 \\ (1,1,1,0) & 1 & 0 & 0 & 0 & 1 & 0 & 0 & 0 & 0 & 0 & 0 \\ (1,1,1,1) & 1 & 0 & 0 & 0 & 0 & 0 & 0 & 0 & 0 & 0 & 0\end{array}\right)$

Figure 1: The design matrix $X$ of the model in Example 2.1.

with 11 points. For instance the fraction

$$
\begin{array}{r}
\mathcal{F}_{1}=\{(0,0,0,0),(0,0,0,1),(0,0,1,1),(0,1,0,1),(0,1,1,0),(1,0,0,1), \\
(1,0,1,0),(1,1,0,0),(1,1,0,1),(1,1,1,0),(1,1,1,1)\}
\end{array}
$$

is saturated while

$$
\begin{array}{r}
\mathcal{F}_{2}=\{(0,0,0,0),(0,0,0,1),(0,0,1,1),(0,1,0,0),(0,1,1,0),(1,0,0,1), \\
(1,0,1,0),(1,1,0,0),(1,1,0,1),(1,1,1,0),(1,1,1,1)\}
\end{array}
$$

is not. A direct computation shows that there are $\left(\begin{array}{l}16 \\ 11\end{array}\right)=4,368$ fractions with 11 points: among them 3,008 are saturated, and the remaining 1,360 are not.

\section{Designs and contingency tables with Algebraic Statistics}

As mentioned in the Introduction, we identify a factorial design with a contingency table whose entries are the indicator function of the fraction. In the previous example, $\mathcal{F}_{1}$ is also a $2^{4}$ table with 1 in the cells identified by the point coordinates of $\mathcal{F}_{1}$ and 0 otherwise as in Table 11. To avoid 


\begin{tabular}{|c|c|c|c|c|c|c|}
\hline \multirow{6}{*}{$N\left(\mathcal{F}_{1}\right)$} & & & \multicolumn{2}{|c|}{$A_{1}=0$} & \multicolumn{2}{|c|}{$A_{1}=1$} \\
\hline & & & $A_{2}=0$ & $A_{2}=1$ & $A_{2}=0$ & $A_{2}=1$ \\
\hline & \multirow{2}{*}{$A_{3}=0$} & $A_{4}=0$ & 1 & 1 & 0 & 1 \\
\hline & & $A_{4}=1$ & 1 & 0 & 1 & 1 \\
\hline & \multirow{2}{*}{$A_{3}=1$} & $A_{4}=0$ & 0 & 1 & 1 & 1 \\
\hline & & $A_{4}=1$ & 1 & 0 & 0 & 1 \\
\hline \multirow{6}{*}{$N\left(\mathcal{F}_{2}\right)$} & & & & $=0$ & & $=1$ \\
\hline & & & $A_{2}=0$ & $A_{2}=1$ & $A_{2}=0$ & $A_{2}=1$ \\
\hline & \multirow{2}{*}{$A_{3}=0$} & $A_{4}=0$ & 1 & 0 & 0 & 1 \\
\hline & & $A_{4}=1$ & 1 & 1 & 1 & 1 \\
\hline & \multirow{2}{*}{$A_{3}=1$} & $A_{4}=0$ & 0 & 1 & 1 & 1 \\
\hline & & $A_{4}=1$ & 1 & 0 & 0 & 1 \\
\hline
\end{tabular}

Table 1: 4-way contingency tables $N\left(\mathcal{F}_{1}\right)$ and $N\left(\mathcal{F}_{2}\right)$ from Example 2.1.

misunderstandings, $\mathcal{F}$ denotes the fraction, while $N(\mathcal{F})$ denotes the corresponding binary table. In Table 1 the contingency table representations of the fractions $\mathcal{F}_{1}$ and $\mathcal{F}_{2}$ from Example 2.1 are displayed.

Such an identification leads us to use the Algebraic Statistics tools from both contingency tables and Design of Experiments theories.

In order to give a complete account of our theory and to present our algorithm with full details, some definitions and a few basic facts concerning the algebraic representation of contingency tables and the combinatorial properties of some statistical models are reported. For the Algebraic Statistics notions, the reader can refer to [10] or [17]. For details on Algebraic notions, see [7] and [14,

Given a contingency table with $K$ cells, we consider the polynomial ring $\mathbb{R}[x]=\mathbb{R}\left[x_{1}, \ldots, x_{K}\right]$ of all polynomials with indeterminates $x_{1}, \ldots, x_{K}$ and real coefficients. The relevant polynomial ring has one indeterminate for each cell of the table (or equivalently, for each point of the design). An ideal $\mathcal{I}$ in $\mathbb{R}[x]$ is a subset of $\mathbb{R}[x]$ such that $f+g \in \mathcal{I}$ for all $f, g \in \mathcal{I}$ and $f g \in \mathcal{I}$ for all $f \in \mathcal{I}$ and for all $g \in \mathbb{R}[x]$.

In Algebraic Statistics, a class of ideals is of special interest. Given a $s \times K$ non-negative matrix $A$ with integer entries, the toric ideal defined by $A$ is the binomial ideal

$$
\mathcal{I}_{A}=\left\{x^{a}-x^{b}: A a=A b\right\}
$$

where the monomials $x^{a}$ are written in vector notation $x^{a}=x_{1}^{a_{1}} \cdots x_{K}^{a_{K}}$. 
Thanks to the Hilbert's basis theorem, every ideal has a finite basis $\left\{f_{1}, \ldots, f_{n}\right\}$ : for all $f \in \mathcal{I}$ there are polynomials $g_{1}, \ldots, g_{n} \in \mathbb{R}[x]$ such that $f=g_{1} f_{1}+\cdots+g_{n} f_{n}$. The computation of a system of generators of an ideal is a non trivial task in Computer Algebra. An actual way to do that is to compute the reduced Gröbner basis of the ideal. The computation of a Gröbner basis depends on the term-order chosen in the polynomial ring $\mathbb{R}[x]$, but for a given term-order the reduced Gröbner basis is unique and can be computed through symbolic software.

Among all term-orders, the elimination term-order for a given indeterminate, say $x_{K}$, leads to the Gröbner basis of the projection $\operatorname{Elim}\left(x_{K} ; \mathcal{I}\right):=$ $\mathcal{I} \cap \mathbb{R}\left[x_{1}, \ldots, x_{K-1}\right]$, just taking the Gröbner basis of $\mathcal{I}$ and removing the polynomials involving $x_{K}$. Applying this fact iteratively, Theorem 4 in [20] shows that the statistical counterpart of elimination of indeterminates is the definition of a statistical model for incomplete tables.

As there are finitely many term-orders, there are finitely many Gröbner bases.

Definition 3.1. Let $\mathcal{I}_{A}$ be a toric ideal. The union of all reduced Gröbner bases of $\mathcal{I}_{A}$ is called the Universal Gröbner basis $\mathcal{U}_{A}$ of $\mathcal{I}_{A}$.

The computation of the Universal Gröbner basis is unfeasible for most ideals, but fortunately there are special algorithms for doing that in the case of toric ideals.

There are now several computer systems for handling multivariate polynomials, see for instance [6] and 8], and all of them compute Gröbner bases. Recently, also the R package mpoly has been implemented, see [13]. For toric ideals the fastest algorithms are implemented in 4ti2, see [26].

Together with the Universal Gröbner basis, there are other combinatorial and polynomial objects derived from a nonnegative integer matrix $A$.

Definition 3.2. A binomial $f=x^{a}-x^{b} \in \mathcal{I}_{A}$ is primitive if there is no binomial $g=x^{c}-x^{d} \in \mathcal{I}_{A}$, with $g \neq f$, such that $c \leq a$ and $d \leq b$. The Graver basis $G r_{A}$ of $A$ is the set of all primitive binomials in $\mathcal{I}_{A}$.

Definition 3.3. The support of a binomial $f=x^{a}-x^{b}$ is the set of indices $i(i=1, \ldots, K)$ such that $a(i) \neq 0$ or $b(i) \neq 0$. We denote the support of $f$ with $\operatorname{supp}(f)$.

Definition 3.4. An irreducible binomial $f=x^{a}-x^{b} \in \mathcal{I}_{A}$ is a circuit if there is no other binomial $g \in \mathcal{I}_{A}$ such that $\operatorname{supp}(g) \subset \operatorname{supp}(f)$ and $\operatorname{supp}(g) \neq \operatorname{supp}(f)$. We denote the set of all circuits of $\mathcal{I}_{A}$ with $\mathcal{C}_{A}$. 
It is easy to see that every circuit is primitive. Moreover, Theorem 1.1 in [15] states that

$$
\mathcal{C}_{A} \subseteq \mathcal{U}_{A} \subseteq G r_{A}
$$

We will come back on such inclusions later in Section 5 .

Remark 3.1. The notion of Universal Markov basis in Statistics for contingency tables has been already introduced in [21] and [22] for the analysis of bounded tables. The circuits are shown to be relevant objects for finding all the supports of the probability distributions in the closure of an exponential family for finite sample spaces, see [23].

\section{Characterization of saturated designs}

We are now ready to state the main result, exploiting the connections between the saturated fractions and the circuits of the design matrix. Recall that the support of a binomial $f=x^{u}-x^{v}$ is the set of indices for which $u>0$ or $v>0$.

In the algebraic theory of toric ideals it is common to use the transpose of the design matrix $X$ in place of the design matrix $X$. In order to simplify the notation, we denote by $A=X^{t}$ the transpose of the design matrix, and with a slight abuse of notation we call it design matrix. As a consequence, given $\mathcal{F}=\left\{i_{1}, \ldots, i_{p}\right\}, A_{\mathcal{F}}$ is the submatrix of $A$ obtained by selecting the columns of $A$ according to $\mathcal{F}$. Note that each column of $A$ identifies a design point, and therefore the definition of a set of column-indices is equivalent to the definition of the fraction with the corresponding design points.

Theorem 4.1. Let $A$ be a full-rank design matrix with dimensions $p \times K$ and let $\mathcal{C}_{A}=\left\{f_{1}, \ldots, f_{L}\right\}$ be the set of its circuits. Given a set $\mathcal{F}$ of $p$ column-indices of $A$, the submatrix $A_{\mathcal{F}}$ is non-singular if and only if $\mathcal{F}$ does not contains any of the supports $\operatorname{supp}\left(f_{1}\right), \ldots, \operatorname{supp}\left(f_{L}\right)$.

Proof. We prove that $A_{\mathcal{F}}$ is singular if and only if there is a binomial $f$ in $\mathcal{C}_{A}$ with $\operatorname{supp}(f) \subseteq \mathcal{F}$.

" $\Rightarrow$ ": If $A_{\mathcal{F}}$ is singular, then there is a null linear combination of its columns. As the entries of $A_{\mathcal{F}}$ are nonnegative integers, the linear combination has coefficients in $\mathbb{Q}$, and hence there is a linear combination with coefficients in $\mathbb{Z}$.

Therefore, there exists a non-zero vector $m \in \mathbb{Z}^{p}$ with $A_{\mathcal{F}} m=0$. Using the positive part of $m, m^{+}=\max \{m, 0\}$, and the negative part $m^{-}=$ 
$-\min (m, 0)$, we decompose $m=m^{+}-m^{-}$, and the binomial $x^{m^{+}}-x^{m^{-}}$ belongs to $\mathcal{I}_{A_{\mathcal{F}}}$ the toric ideal associated to $A_{\mathcal{F}}$.

As $\mathcal{I}_{A_{\mathcal{F}}}$ can be computed from $\mathcal{I}_{A}$ with the elimination algorithm as described in Section 3, and in particular $\mathcal{I}_{A_{\mathcal{F}}}=\operatorname{Elim}\left(x_{i}: i=1, \ldots, K, i \notin\right.$ $\left.\mathcal{F} ; \mathcal{I}_{A}\right), \mathcal{I}_{A_{\mathcal{F}}}$ is non-empty and there is a binomial in the Gröbner basis $\mathcal{G}_{A, \tau}$, where $\tau$ is the elimination term order for the indeterminates $\left(x_{i}: i=\right.$ $1, \ldots, K, i \notin \mathcal{F})$. By definition of Universal Gröbner basis, this implies that there is a binomial $g$ in $\mathcal{U}_{A}$ with support in $\mathcal{F}$.

If the binomial $g$ is a circuit, the proof if complete. If not, there do exist a circuit $h \in \mathcal{C}_{A}$ with $\operatorname{supp}(h) \subset \operatorname{supp}(g)$ and it is enough to choose such binomial $h$.

" $\Leftarrow$ ": Suppose that there is a circuit $f \in \mathcal{C}_{A}$ with support in $\mathcal{F}$, i.e., $x^{m^{+}}-x^{m^{-}}$is in $\mathcal{I}_{A}$. Hence, $A m=0$ and projecting onto the subspace $\mathbb{R}\left[x_{i}: i \in \mathcal{F}\right]$ we obtain $A_{\mathcal{F}} m=0$, i.e., $A_{\mathcal{F}}$ is singular.

Theorem 4.1 replaces a linear algebra condition (namely the non-singularity of the design matrix) with a combinatorial property for checking weather a fraction is saturated or not. This technique highlight an interesting combinatorial property of the saturated fractions, with a theoretical interest. It may be of limited practical interest when analyzing a single fraction, but it becomes useful when we need to study all the saturated fractions of a given factorial design. Note that the set of the circuits $\mathcal{C}_{A}$ of a design matrix can be computed once for all fractions.

We are now ready to analyze and discuss some examples. To ease the presentation, the binomials are defined through their exponents, so that a nonnegative vector $a-b$ is used in place of $x^{a}-x^{b}$. For instance the binomial $x_{1} x_{3}^{2}-x_{2} x_{7} x_{8}$ in $\mathbb{R}\left[x_{1}, \ldots, x_{8}\right]$ is written as $(1,-1,2,0,0,0,-1,-1)$. This notation is the standard one in 4ti2, the software we use for our computations.

Example 4.1. We consider again the $2^{4}$ design and the model with simple effects and 2-way interactions, already discussed in Example 2.1. In less than one second, 4ti2 produces the list of all 140 circuits of the design matrix. Labeling the design points lexicographically, the 140 circuits can be divided into three classes, up to permutations of factors or levels:

- 20 circuits of the form

$$
f_{1}=(0,0,0,0,1,-1,-1,1,-1,1,1,-1,0,0,0,0)
$$

with $\max \left|f_{1}\right|=1$; 


\begin{tabular}{|c|c|c|c|c|c|}
\hline & & \multicolumn{2}{|c|}{$A_{1}=0$} & \multicolumn{2}{|c|}{$A_{1}=1$} \\
\hline & & $A_{2}=0$ & $A_{2}=1$ & $A_{2}=0$ & $A_{2}=1$ \\
\hline \multirow{2}{*}{$A_{3}=0$} & $A_{4}=0$ & & $\bullet$ & $\bullet$ & \\
\hline & $A_{4}=1$ & & $\bullet$ & $\bullet$ & \\
\hline \multirow{4}{*}{$A_{3}=1$} & $A_{4}=0$ & & • & $\bullet$ & \\
\hline & $A_{4}=1$ & & - & • & \\
\hline & & & $=0$ & $A_{1}$ & $=1$ \\
\hline & & $A_{2}=0$ & $A_{2}=1$ & $A_{2}=0$ & $A_{2}=1$ \\
\hline \multirow{2}{*}{$A_{3}=0$} & $A_{4}=0$ & $\bullet$ & & & $\bullet$ \\
\hline & $A_{4}=1$ & • & $\bullet$ & & \\
\hline \multirow{4}{*}{$A_{3}=1$} & $A_{4}=0$ & & • & $\bullet$ & $\bullet$ \\
\hline & $A_{4}=1$ & $\bullet$ & & & • \\
\hline & & \multicolumn{2}{|c|}{$A_{1}=0$} & \multicolumn{2}{|c|}{$A_{1}=1$} \\
\hline & & $A_{2}=0$ & $A_{2}=1$ & $A_{2}=0$ & $A_{2}=1$ \\
\hline \multirow{2}{*}{$A_{3}=0$} & $A_{4}=0$ & $\bullet$ & & $\bullet$ & $\bullet$ \\
\hline & $A_{4}=1$ & & • & $\bullet$ & \\
\hline \multirow{2}{*}{$A_{3}=1$} & $A_{4}=0$ & & • & $\bullet$ & $\bullet$ \\
\hline & $A_{4}=1$ & $\bullet$ & & & • \\
\hline
\end{tabular}

Table 2: The supports of the three circuits in Example 4.1. The bullet symbol denotes the cells in the support

- 40 circuits of the form

$$
f_{2}=(1,-2,0,1,0,1,-1,0,0,1,-1,0,-1,0,2,-1)
$$

with $\max \left|f_{2}\right|=2$;

- 80 circuits of the form

$$
f_{3}=(1,0,-2,1,0,-1,1,0,-2,1,3,-2,1,0,-2,1)
$$

with $\max \left|f_{3}\right|=3$.

The supports of these circuits are displayed in Figure 2. Note that the support of $f_{2}$ is contained in the fraction $\mathcal{F}_{2}$ of Example [2.1, making that fraction non-saturated.

Remark 4.1. Theorem 4.1 allows us to identify saturated designs with the feasible solutions of an integer linear programming problem. Let $\overline{\mathcal{C}}_{A}=$ $\left(c_{i j}, i=1, \ldots, L, j=1, \ldots, K\right)$ be the $L \times K$ matrix, whose rows contain the 
values of the indicator functions of the supports of the circuits $f_{1}, \ldots, f_{L}$, $c_{i j}=\left(f_{i j} \neq 0\right), i=1, \ldots, L, j=1, \ldots, K$ and $Y=\left(y_{1}, \ldots, y_{K}\right)$ be the $K$-dimensional column vector that contains the unknown values of the indicator function of the points of $\mathcal{F}$. The vector $Y$ must satisfy the following conditions:

$$
\begin{gathered}
\overline{\mathcal{C}}_{A} Y<b, \\
1_{K}^{t} Y=p, \\
y_{i} \in\{0,1\}
\end{gathered}
$$

where $b=\left(b_{1}, \ldots, b_{L}\right)$ is the column vector defined by $b_{i}=\# \operatorname{supp}\left(f_{i}\right), i=$ $1, \ldots, L$, and $1_{K}$ is the column vector of length $K$ and whose entries are all equal to 1.

\section{Computational remarks}

We have introduced in Section 3 three different objects associated with the design matrix of a model, namely the circuits, the Graver basis and the Universal Gröbner basis. In general strict inclusions among them holds, as stated in Eq. (2). Therefore, it is interesting to find models for which such three sets coincide. For instance, one may have the Graver basis theoretically determined, and in such case no further computations are needed.

The basic definition for investigating this issue is the following one.

Definition 5.1. A nonnegative integer matrix with rank $p$ is unimodular if all its non-zero $p \times p$ minors are equal to \pm 1 . A nonnegative integer matrix is totally unimodular if all its non-zero minors are equal to \pm 1 .

Of course, a totally unimodular matrix is unimodular. It follows immediately from Definition 5.1 that the entries of a totally unimodular matrix are 0 and 1, that each submatrix of a totally unimodular matrix is again totally unimodular, and the transpose of a totally unimodular matrix is again totally unimodular. A couple of less intuitive properties are collected in the following proposition.

Proposition 5.1. Let $A$ be a $0-1$ matrix with dimensions $p \times K$.

1. If for each subset $J$ of columns of $A$, there is a partition $\left\{J_{1}, J_{2}\right\}$ of $J$ such that

$$
\left|\sum_{j \in J_{1}} A_{i, j}-\sum_{j \in J_{2}} A_{i, j}\right| \leq 1 \text { for } i=1, \ldots, p,
$$


then $A$ is totally unimodular. In particular, if each row contains at most 2 non-zero entries, then $A$ is totally unimodular.

2. All matrices obtained by pivot operations on a totally unimodular matrix are totally unimodular.

For the theory of totally unimodular matrices, the reader can refer to [24], Chapters 19 and 20. The following result can be found in [25], page 70.

Proposition 5.2. If $A$ is a unimodular matrix, then

$$
\mathcal{C}_{A}=\mathcal{U}_{A}=G r_{A}
$$

In view of above properties, we study the first non-trivial model, i.e., the no- $d$-way interaction model for $d$ factors. All other model matrices are submatrices of this matrix. For $d=2$, we reduce to the independence model, and it is known that the design matrix of the independence model is totally unimodular for arbitrary $s_{1}$ and $s_{2}$. Indeed, an alternative parametrization of the no-2-way interaction for two factors uses the following design matrix

$$
\tilde{X}=\left(a_{0}|\ldots| a_{s_{1}-1}\left|b_{0}\right| \ldots \mid b_{s_{2}-2}\right),
$$

in place of the design matrix in Eq. (11) discussed in Section 2, $\tilde{X}$ satisfies the hypotheses of the first item in Proposition 5.1. To move to higher dimensions, we need to work with Lawrence liftings. Given a matrix $A$, its Lawrence lifting is the block matrix defined by

$$
\Lambda(A)=\left(\begin{array}{cc}
A & I \\
0 & I
\end{array}\right)
$$

where $I$ is the identity matrix and 0 is a null matrix with suitable dimensions.

As argued in 25], the Lawrence lifting of a totally unimodular matrix is totally unimodular as well, and in [15], Section 4.1 is proved the following fact: given a no- $d$-way interaction model for a $s_{1} \times \cdots \times s_{d}$ design with design matrix $A$, the no- $(d+1)$-interaction model for the $s_{1} \times \cdots \times s_{d} \times 2$ is the Lawrence lifting $\Lambda(A)$ of $A$. This property is derived with details in [16].

Combining all the facts in the discussion above, the following theorem is proved.

Theorem 5.1. The design matrix of all models for a $s_{1} \times s_{2} \times 2 \times \cdots \times 2$ factorial designs is totally unimodular. 
The simplest model with a non unimodular design matrix is the no-3way interaction model for the $3 \times 3 \times 3$ factorial design. Under the name of "transportation problem", this model is fully discussed in [25], page 150 . However in this case the circuits and the Graver basis still coincide. The simplest model where circuits and Graver basis differ is the no-3-way interaction model for the $3 \times 3 \times 4$ factorial design. The results are presented in the next section.

To compute the circuits and the Graver basis of our running example and of the examples in the next section, we used the commands circuits and graver in 4ti2, 26]. All computations were carried out in less than 2 seconds on a standard PC. As usual in Computer Algebra, the computational complexity increases quickly as the number of indeterminates rises and it depends heavily on the degrees of freedom of the model. For instance, the design $2^{5}$ under the model with simple effects and 2-way interactions is a computationally unfeasible problem on a standard PC.

Finally, notee that the elements of the bases (Graver or circuits) are the coefficients of the linear combination of fraction points to have zero as result, and therefore a singular matrix. Therefore, the elements of the bases with more than $p$ values different from zero are not of interest of our aims. In our running examples, the circuits of the third kind like the circuit $f_{3}$ in Eq. (5) can be excluded, and in the following examples this situation comes up in other few cases.

\section{Examples}

In this section we briefly describe the results of our computations for some classical models.

- Design $2^{5}$; model with simple factors and 2-way and 3-way interactions.

The saturated model has 26 points. Circuit basis and Graver basis are equal. They contain 3,254 elements that can be divided into 12 classes, up to permutations of factors or levels. All of them have support cardinality less than 26 (and the maximum cardinality is just 26).

- Design $2^{5}$; model with simple factors.

The saturated model has 6 points. Also in this case circuit basis and Graver basis are equal. The circuits are 353, 616 elements that can be divided into 38 classes, up to permutations of factors or levels. There 
are 259, 904 circuits with support cardinality equal to 7 , and therefore the circuits to be checked in our algorithm are 93,712 with support cardinality ranging from 4 to 6 .

- Design $2 \times 3 \times 4$; model with simple factors and 2-way interactions.

The saturated model has 18 points. Circuit basis and Graver basis are equal, and they contain 42 elements that can be divided into two classes. One class of 24 elements has support cardinality 12, and another class of 18 elements has support cardinality 8. All of them are needed for the algorithm.

- Design $3 \times 3 \times 4$; model with simple factors and 2-way interactions.

The saturated model has 24 points. Circuit basis and Graver basis are different. The latter has 19,722 elements that can be divided into 20 classes, while the former has 17,994 elements with 19 classes, all included in the Graver basis. Both bases contain 15, 302 elements with support cardinality smaller than or equal to 24 . A configuration in the Graver basis which is not a circuit is:

$$
\begin{aligned}
(-1,1,1-1,1,0,0,-1,0,-1,-1,2,0,-1,0,1,-1,2,-1 \\
0,1,-1,1,-1,1,0,-1,0,0,-2,1,1,-1,2,0,-1)
\end{aligned}
$$

All the elements in the Graver basis but not in the circuit basis are permutations of this configuration.

\section{Generation of random saturated fractions}

When a sequence of saturated fractions is needed, instead of a single saturated fraction, an algorithm for finding such fractions without computing the determinant of the design matrix of each fraction may be useful.

As a first application, one can generate random fractions with $p$ points and then check whether they are saturated or not simply by comparing the selected fractions with the circuit basis. For instance, we have implemented that procedure for the model in our running example, and we are able to generate 5,000 random saturated fractions in about 1 second on a standard PC, by executing few lines of code. For our simulations, we used R, see [18].

However, it is interesting to take a deeper look into the connections between Markov bases and saturated fractions. Therefore, we show how to apply the theory of Markov bases to generate saturated fractions with given projections. Indeed, the combinatorial objects needed to check whether a 
fraction is saturated or not are essentially the same as those needed to define a Markov Chain Monte Carlo sampler.

Remember that we have already identified a fraction with a binary contingency table. Given a fraction $\mathcal{F}$, the corresponding table is $N(\mathcal{F})$, where $N(\mathcal{F})_{i_{1}, \ldots, i_{d}}=1$ if $\left(i_{1}, \ldots, i_{d}\right)$ is a point of $\mathcal{F}$ and $N(\mathcal{F})_{i_{1}, \ldots, i_{d}}=0$ otherwise. Using Algebraic statistics tools, we are able to generate all fractions with given margins, or projections, through a Markov chain algorithm following the theory in [21]. Moreover, we can merge that algorithm with our theory in order to select only the saturated fractions with given margins. Interestingly, the Algebraic and Combinatorial objects needed to define the relevant Markov chain for navigating the set of all fractions with given margins and for checking whether the fraction is saturated are very close.

Basic facts about Markov bases are reported. The reader can refer to the book [10] for a complete presentation. A Markov move $m$ is a table with integer entries such that $N(\mathcal{F}), N(\mathcal{F})+m$ and $N(\mathcal{F})-m$ have the same margins. A Markov basis $\mathcal{M}$ is a finite set of Markov moves which makes connected the set of all tables, or designs, with the same margins. Notice that by adding Markov moves to a Markov basis yields again a Markov basis.

In standard problems involving contingency tables, Theorem 3.1 in [9] states that an actual way for writing a Markov basis of a design matrix $A$ is as follows. Compute a Gröbner basis of the toric ideal $\mathcal{I}_{A}$ (w.r.t. an arbitrary term-order) and then define the moves by taking the logarithms of the binomials $\left(p^{a}-p^{b} \mapsto a-b\right)$ in the Gröbner basis.

As observed in [21] and [22], when the cells of the table are bounded, we need a special Markov basis, namely the Universal Markov basis, derived from the Universal Gröbner basis and taking logarithms. In our problem, the relevant tables are bounded as each entry can be only 0 or 1 . Therefore, in what follows we consider the moves of the Universal Markov basis.

If we start from a fraction with matrix $N\left(\mathcal{F}_{0}\right)$, the Markov chain is then built as follows:

- at each step $i$, randomly choose a Markov move $m$ in $\mathcal{M}$ and a sign $\varepsilon \in\{ \pm 1\}$

- if $N\left(\mathcal{F}_{i}\right)+\varepsilon m$ is a binary table, move the chain to $N\left(\mathcal{F}_{i+1}\right)=N\left(\mathcal{F}_{i}\right)+$ $\varepsilon m$; otherwise, stay in $N\left(\mathcal{F}_{i}\right)$.

The Markov chain described above is a connected chain over all the designs with fixed margins, and its stationary distribution is the uniform one. By considering the classical Metropolis-Hastings probability ratio, one can define a Markov chain converging to any specified probability distribution, see 
[9].

Now, a set of saturated fractions can be extracted from the Markov chain above, by comparing each fraction with the supports of the relevant circuits, as described in Section 4 , and discarding the non-saturated fractions.

Two computational remarks are now in order: (a) the Universal Gröbner basis coincide with the set of circuits, and the Universal Markov basis does not need new computations; (b) due to the limitation to $0-1$ tables, we can discard all the moves with values outside $\{-1,0,1\}$ as they do not produce valid tables in the algorithm above.

Example 7.1. We now reconsider the $2^{4}$ design with simple effects and 2way interactions, already illustrated in the previous sections. Starting from the fraction $\mathcal{F}$ in Example 2.1, we are interested in saturated fractions with the same one-way projections. The Universal Gröbner basis for this problem coincides with the circuits basis and consists of 1,348 elements, 532 of them have values in $\{-1,0,1\}$ and are needed to define the Markov chain. Taking such set of moves as input, we are able to produce a sequence of fractions with the same one-way projections as $\mathcal{F}_{1}$. Comparing each fraction with the supports of the 120 circuits found in Example [2.1, we have a random sample of saturated fractions. In less than 1 minute, the execution of a simple $\mathrm{R}$ function yields a sample of 5,000 saturated fractions with the desired projection.

\section{Conclusions}

The theory described in this paper suggests several extensions and applications. Firstly, it is interesting to explore how the results can be extended for the characterization of saturated fractions to more general designs.

Secondly, the connections between fractions and graphs need to be studied. Indeed, it is known that the circuits for two-factor designs can be derived by the complete bipartite graph associated with the design, but little is known in the general case.

It would also be interesting to study the classification of the saturated fractions with respect to some statistical criteria. Among these criteria, we cite the minimum aberration in a classical sense, or more recent tools, such as state polytopes. Minimum aberration is a classical notion in this framework, and is supported by a large amount of literature. This theory has been developed in [12, and more recently in, e.g., [27] with the use of the indicator function in the two-level case. The extension to the multilevel 
case is currently an open problem. The use of state polytopes has been introduced in [5].

For applications, it would be interesting to define an algorithm to optimize the search of saturated fractions using the circuit basis in the construction of the fractions instead of checking a random fraction.

Finally, the use of the inequivalent saturated fractions to perform exact tests on model parameters is worth studying, together with its implementation in statistical softwares, such as SAS or R. For inequivalent orthogonal arrays very interesting results have already been achieved, see [4] and [2].

\section{References}

[1] Aoki, S., Takemura, A.: Markov chain Monte Carlo tests for designed experiments. J. Statist. Plann. Inference 140(3), 817-830 (2010)

[2] Arboretti Giancristofaro, R., Fontana, R., Ragazzi, S.: Construction and nonparametric testing of orthogonal arrays through algebraic strata and inequivalent permutation matrices. Communications in Statistics - Theory and Methods 41(16-17) (2012). DOI 10.1080/03610926.2011. 579380

[3] Bailey, R.A.: Design of comparative experiments. Cambridge Series in Statistical and Probabilistic Mathematics. Cambridge University Press, Cambridge (2008)

[4] Basso, D., Salmaso, L., Evangelaras, H., Koukouvinos, C.: Nonparametric testing for main effects on inequivalent designs. In: mODa $7-$ Advances in model-oriented design and analysis, Contrib. Statist., pp. 33-40. Physica, Heidelberg (2004)

[5] Berstein, Y., Maruri-Aguilar, H., Onn, S., Riccomagno, E., Wynn, H.: Minimal average degree aberration and the state polytope for experimental designs. Ann. Inst. Statist. Math. 62(4), 673-698 (2010). DOI $10.1007 / \mathrm{s} 10463-010-0291-8$

[6] CoCoATeam: CoCoA: a system for doing Computations in Commutative Algebra. Available at http://cocoa.dima.unige.it (2010)

[7] Cox, D., Little, J., O'Shea, D.: Ideals, Varieties, and Algorithms. Springer Verlag, New York (1992) 
[8] Decker, W., Greuel, G.M., Pfister, G., Schönemann, H.: Singular 31-6 - A computer algebra system for polynomial computations (2012). Http://www.singular.uni-kl.de

[9] Diaconis, P., Sturmfels, B.: Algebraic algorithms for sampling from conditional distributions. Ann. Statist. 26(1), 363-397 (1998)

[10] Drton, M., Sturmfels, B., Sullivant, S.: Lectures on Algebraic Statistics. Birkhauser, Basel (2009)

[11] Fontana, R., Rapallo, F., Rogantin, M.P.: Markov bases for sudoku grids. In: A. Di Ciaccio, M. Coli, J.M. Angulo Ibanez (eds.) Advanced Statistical Methods for the Analysis of Large Data-Sets, Studies in Theoretical and Applied Statistics, pp. 305-315. Springer, Berlin (2012)

[12] Fries, A., Hunter, W.G.: Minimum aberration $2^{k-p}$ designs. Technometrics 22(4), 601-608 (1980). DOI 10.2307/1268198

[13] Kahle, D.: mpoly: Symbolic computation and more with multivariate polynomials (2013). URL http://CRAN.R-project.org/package=mpoly. R package version 0.0 .3

[14] Kreuzer, M., Robbiano, L.: Computational Commutative Algebra 1. Springer, New York (2000)

[15] Ohsugi, H.: A dictionary of Gröbner bases of toric ideals. In: T. Hibi (ed.) Harmony of Gröbner Bases and the Modern Industrial Society, pp. 253-281. World Scientific, Singapore (2012)

[16] Ohsugi, H., Hibi, T.: Non-very ample configurations arising from contingency tables. Ann. Inst. Statist. Math. 62(4), 639-644 (2010). DOI 10.1007/s10463-010-0288-3

[17] Pistone, G., Riccomagno, E., Wynn, H.P.: Algebraic Statistics: Computational Commutative Algebra in Statistics. Chapman\&Hall/CRC, Boca Raton (2001)

[18] R Development Core Team: R: A Language and Environment for Statistical Computing. R Foundation for Statistical Computing, Vienna, Austria (2011). URL http://www.R-project.org/

[19] Raktoe, B.L., Hedayat, A., Federer, W.T.: Factorial designs. Wiley Series in Probability and Mathematical Statistics. John Wiley \& Sons Inc., New York (1981) 
[20] Rapallo, F.: Markov bases and structural zeros. J. Symbolic Comput. 41(2), 164-172 (2006)

[21] Rapallo, F., Rogantin, M.P.: Markov chains on the reference set of contingency tables with upper bounds. Metron 65(1), 35-51 (2007)

[22] Rapallo, F., Yoshida, R.: Markov bases and subbases for bounded contingency tables. Ann. Inst. Statist. Math. 62(4), 785-805 (2010)

[23] Rauh, J., Kahle, T., Ay, N.: Support sets in exponential families and oriented matroid theory. Internat. J. Approx. Reason. 52(5), 613-626 (2011). DOI 10.1016/j.ijar.2011.01.013

[24] Schrijver, A.: Theory of Linear and Integer Programming. John Wiley \& Sons, New York (1986)

[25] Sturmfels, B.: Gröbner bases and convex polytopes, University lecture series (Providence, R.I.), vol. 8. American Mathematical Society (1996)

[26] 4ti2 team: 4ti2 - a software package for algebraic, geometric and combinatorial problems on linear spaces. Available at www.4ti2.de (2008)

[27] Ye, K.Q.: Indicator function and its application in two-level factorial designs. Ann. Statist. 31(3), 984-994 (2003). DOI 10.1214/aos/ 1056562470

Acknowledgment. RF acknowledges SAS institute for providing software. FR is partially supported by the PRIN2009 grant number 2009H8WPX5. 